PANCREAS

\title{
Antibodies to carbonic anhydrase and lgG4 levels in idiopathic chronic pancreatitis: relevance for diagnosis of autoimmune pancreatitis
}

\author{
L Aparisi, A Farre, L Gomez-Cambronero, J Martinez, G De Las Heras, J Corts, S Navarro, J Mora, \\ M Lopez-Hoyos, L Sabater, A Ferrandez, D Bautista, M Perez-Mateo, S Mery, J Sastre
}

Gut 2005;54:703-709. doi: 10.1136/gut.2004.047142

See end of article for authors' affiliations

Correspondence to:

Dr L Aparisi, Servicio de Hepatología, Hospital Clínico Universitario, Avda. Blasco lbañez 17, 46010 Valencia, Spain; aparisi_lui@gra.es

Revised version received 5 October 2004 Accepted for publication 12 October 2004
Background: Increased serum antibodies against carbonic anhydrase II (CA-II Ab) or lgG4 levels have been reported in cases of autoimmune chronic pancreatitis (ACP).

Aim: To assess the relevance of serum CA-II Ab and lgG4 levels for the diagnosis of ACP in idiopathic CP (ICP) versus alcoholic CP and Siögren's syndrome (SS).

Subjects: This was a multicentre study involving 227 subjects divided into four groups: ICP $(n=54)$, normal controls ( $n=54$, paired by age and sex with ICP patients), alcoholic CP $(n=86)$, and SS $(n=33)$.

Methods: CA-II Ab was measured by ELISA and confirmed by western blotting. A score of easy clinical use with major clinical, morphological, and biochemical parameters for the diagnosis of ACP was applied. Results: The percentage of patients with increased serum CA-II Ab was higher in the ICP group (28\%) than in controls (1.9\%) and in patients with alcoholic CP (10.5\%), but lower than in patients with SS (64\%). The proportion with elevated lgG4 levels was higher in the ICP group (15\%) compared with controls (1.9\%) and SS (0\%) but not significantly different from alcoholic CP (8\%). Most ICP patients (7/8) with high lgG4 levels exhibited increased CA-II Ab and a compatible ACP score. A definitive diagnosis of ACP by histological analysis was associated with other autoimmune disorders, an increase in both serum lgG4 and CA-II Ab levels, and IgG4 positive plasma cells.

Conclusions: The increase in serum lgG4 levels was strongly associated with elevated CA-II Ab levels, manifestations compatible with $\mathrm{ACP}$, and lymphoplasmacytic infiltration when surgical specimens were available.
$\Lambda$ utoimmunity is considered an aetiological factor for numerous exocrine gland diseases, such as autoimmune pancreatitis (ACP) and Sjögren's syndrome (SS). ${ }^{12}$ It was suggested that these diseases affect different organs through an autoimmune reaction against a common antigen, ${ }^{3}$ which should be expressed in epithelial cells from ducts of exocrine glands. Carbonic anhydrase II (CA-II) may be one of these common antigens as autoantibodies against CA-II have been found in the serum of patients with SS, systemic lupus erythematosus, autoimmune cholangitis, and chronic pancreatitis (CP). ${ }^{3-6}$ Moreover, immunisation with CA-II leads to sialoadenitis, cholangitis, or pancreatitis in mice. $^{7-9}$

Idiopathic chronic pancreatitis (ICP) represents approximately $10-30 \%$ of CP cases. A significant proportion of these cases should be ascribed to ACP, which was first described by Sarles and colleagues ${ }^{10}$ in 1961 as a primary inflammatory pancreatitis. Later, Yoshida and colleagues ${ }^{11}$ proposed the term ACP for this disease, which is characterised by increased serum levels of $\gamma$-globulin and/or IgG, presence of autoantibodies, diffuse pancreatic fibrosis with lymphocyte and plasma cell infiltration, and irregular narrowing of the main pancreatic duct. ${ }^{12-15}$

The lack of specific biochemical markers is a major drawback for the diagnosis of ACP. In this regard, Hamano and colleagues ${ }^{14}$ have recently reported that patients with autoimmune (sclerosing) CP exhibit high serum IgG4 levels, thus providing a means of distinguishing this disease from other pancreatic disorders. Furthermore, according to Hamano and colleagues, ${ }^{14}$ the increase in IgG4 serum levels could also distinguish ACP from other autoimmune exocrinopathies, such as SS.
Although CA-II may be considered as a target antigen in the pathophysiology of ACP, ${ }^{3}$ the association of CA antibodies $(\mathrm{Ab})$ with increased IgG4 serum levels for the diagnosis of ACP has not yet been studied. To our knowledge, this is the first multicentre study involving a large number of patients that has assessed the relevance of serum CA-II Ab and IgG4 serum levels for the diagnosis of ACP in ICP versus alcoholic $\mathrm{CP}$ and SS. The association between increased $\mathrm{CA} A \mathrm{~b}$ and IgG4 levels and the clinical manifestations ascribed to ACP were also assessed.

\section{METHODS}

Study subjects

This multicentre study involved 227 subjects from five hospitals. Subjects were classified into four groups: (1) patients with ICP $(\mathrm{n}=54)$; (2) normal subjects as controls $(\mathrm{n}=54)$, age and sex matched with patients from the ICP group; (3) patients with alcoholic CP $(\mathrm{n}=86)$; and (4) patients with SS $(n=33)$. Table 1 shows the general characteristics of these subjects. Diagnosis of ICP and alcoholic CP was performed according to the Cambridge and Marsella criteria. ${ }^{16}{ }^{17}$ Diagnosis of SS was performed according to the criteria established by the specific Study Group from the European Community. ${ }^{18}$ None of the patients with SS included in this study exhibited any pancreatic disorder. The absence of pancreatic disease in these patients was assessed on clinical grounds, pancreatic function-faecal

Abbreviations: $A C P$, autoimmune chronic pancreatitis; $C A-\| \mathrm{Ab}$, antibodies to carbonic anhydrase II; CP, chronic pancreatitis; ELISA, enzyme linked immunosorbent assay; ICP, idiopathic chronic pancreatitis; SS, Sjögren's syndrome; ANA, antinuclear antibodies 
pancreatic El elastase or pancreolauryl test-and morphological analyses by computed tomography or ultrasonography.

\section{Assays}

Serum antinuclear antibodies (ANA) were analysed by enzyme linked immunosorbent assay (ELISA) (DiaSorin srl, Saluggia, Italy) and confirmed by indirect immunofluorescence assay on Hep-2 cell slides (Cormedica, Barcelona, Spain). Serum antimitochondrial and antismooth muscle antibodies were studied by indirect immunofluorescence assay on rat liver/kidney/stomach slides (Biosystems, Barcelona, Spain). Antibodies against Ro (SS-A) and La (SS-B) were detected in serum by the INNO-LIA ANA Update immunoassay (Innogenetics, Ghent, Belgium). Rheumatoid factor was quantified by nephelometry (Dade Behring, Marburg, Germany). Serum levels of CA-II Ab were determined by ELISA, as described by Kino-Ohsaki and colleagues, ${ }^{3}$ and expressed as absorbance units. Western blotting was used to confirm the increased serum levels of CA-II Ab above the cut off value previously established by ELISA. Western blotting was performed using the PhototopeHRP detection kit (Cell Signaling, Beverly, Massachusetts, USA) with chemiluminescence detection. IgG4 and total IgG serum levels were measured by nephelometry (DadeBehring). Cut off values were $1200 \mathrm{mg} / \mathrm{dl}$ for $\mathrm{IgG}$ and $130 \mathrm{mg} / \mathrm{dl}$ for IgG4 and were previously established in healthy subjects from the Universitary Clinic Hospital in Valencia using this commercial kit.

\section{Cut off values for serum levels of $C A-I I A b$}

Serum levels of CA-II Ab were measured in 225 healthy donors from the Blood Transfusion Centre, Valencia, Spain. The distribution of values was normal for CA-II Ab, with Z value of 1.13 using the Komolgorov-Smirnov test. Mean (SD) absorbance value was $0.1119(0.0249)$ for CA II-Ab. Cut off values were established as 2 SDs over the mean value. Thus the cut off value for CA II-Ab was 0.162.

\section{Diagnosis of ACP}

A score was used to identify those patients with a diagnosis compatible with ACP according to the following criteria:

- Clinical manifestations: absence of previous acute attacks of pancreatitis, absence of pancreatic pain, occurrence of cholestasis/jaundice, and presence of associated autoimmune disorders. A value of 1 was considered when at least two of these conditions were fulfilled whereas a value of 0 was considered when one or none of these conditions was fulfilled.

- Morphological parameters: absence of calcifications, absence of pseudocysts, presence of pancreatic "mass forming" or enlarged pancreas, and absence of dilatation of the Wirsung duct. A value of 1 was considered when at least two of these conditions were fulfilled whereas a value of 0 was considered when one or none of these conditions was fulfilled.

- Laboratory parameters: increased serum IgG levels and serum ANA. The presence of at least one of these parameters was given a value of 1 whereas absence of these parameters was given a value of 0 .

A total joint score of 0 or 1 was considered "probably not $\mathrm{ACP}$ ", 2 as "possible ACP", and 3 as "probable ACP".

Surgical specimens were available in 10 cases of ICP to perform histopathological analysis to confirm a definitive diagnosis of ACP if lymphoplasmacytic infiltrate and fibrosis were found. ${ }^{15}$

\section{Immunohistochemistry}

Pancreatic specimens were fixed in 10\% formaldehyde and paraffin embedded tissue blocks were prepared. Immunohistochemical analysis was performed using the anti-IgG4 (The Binding Site, Birmingham, UK) antibody with the avidin-biotin peroxidase complex method. ${ }^{19}$

\section{Statistical analysis}

According to the Komolgorov-Smirnov test, most quantitative variables did not fit a normal distribution and consequently non-parametric tests were used. The Mann-Whitney and Kruskal-Wallis tests were used to compare the distribution of quantitative variables among groups. Qualitative variables were analysed by the $\chi^{2}$ test or Fisher's exact test when appropriate. For comparisons between the control and ICP paired groups, the Wilcoxon and McNemar tests were used. A hierarchical cluster analysis was performed using the total sum of square deviations from the mean of a cluster (Ward's method) and the square Euclidean distance (the sum of the square differences between scores for two cases on all variables) in order to identify patients with a compatible diagnosis of ACP among those with ICP. Cluster analysis is a classification method that establishes a set of clusters whose cases within a cluster are more similar to each other than they are to cases in other clusters. Data were standardised using the $\mathrm{z}$ score method for cluster analysis. A p value $<0.05$ was considered significant.

\section{RESULTS}

Clinical, morphological, biochemical, and haematological characteristics in normal subjects and patients with CP or SS

Table 2 shows the frequency of relevant parameters related to the diagnosis of ACP in the four groups of this study. Acute attacks of pancreatitis and abdominal pain as well as pancreatic calcification and pseudocysts were significantly more frequent in patients with alcoholic CP than in those with ICP. Patients with SS had no acute attacks of

Table 1 General characteristics of normal controls and patients with chronic pancreatitis or Sjögren's syndrome

\begin{tabular}{lllll}
\hline & Controls & ICP & Alcoholic CP & Siögren's syndrome \\
\hline Age & $56.5(12.8-80)$ & $56(12.8-82)$ & $52(35-66.7)$ & $62(36.1-77.3)$ \\
Sex (F/M) & $23 / 31$ & $23 / 31$ & $5 / 81^{* *}+\dagger$ & $32 / 1^{* *}+\dagger$ \\
Duration of disease $(y)$ & 0 & $4(0.6-22.3)$ & $8(0.7-19.7)$ & $7(1-20)$ \\
Diabetes mellitus & $0 \%(0 / 54)$ & $40.7 \%(22 / 54)^{* *}$ & $66.3 \%(57 / 86)^{* *}+\dagger$ & $3 \%(1 / 33)+\dagger$ \\
Steatorrhoea & $0 \%(0 / 54)$ & $35.2 \%(19 / 54)^{* *}$ & $53.5 \%(46 / 86)^{* *} \dagger$ & $0 \%(0 / 33) \dagger \dagger$ \\
\hline
\end{tabular}

Results are expressed as median (5th and 95th percentiles), or as percentage. $\mathrm{CP}$, chronic pancreatitis; ICP, idiopathic chronic pancreatitis

Duration of disease is the time from onset of the disease. Diabetes mellitus diagnosis was established following the WHO criteria. Steatorrhoea was diagnosed when faecal fat excretion was $>7 \mathrm{~g} / 24$ hour in faeces of 72 hours. Statistical significance was as follows: ${ }^{* *} p<0.01$ versus controls; $\uparrow p<0.05, \uparrow+p<0.01$ versus ICP. 
Table 2 Clinical, morphological, biochemical, and haematological characteristics in normal controls and patients with chronic pancreatitis or Siögren's syndrome

\begin{tabular}{lllll}
\hline & Controls & ICP & Alcoholic CP & Siögren's syndrome \\
\hline Attacks of pancreatitis & $0(0 / 54)$ & $33.3(18 / 54)^{* *}$ & $81.4(70 / 86)^{* *}+\dagger$ & $0(0 / 33)+\dagger$ \\
Abdominal pain & $0(0 / 54)$ & $60.4(32 / 54)^{* *}$ & $91.9(79 / 86)^{* *}+\dagger$ & $9.1(3 / 33)^{*}+\dagger$ \\
Cholestasis/jaundice & $0(0 / 54)$ & $22.2(12 / 54)^{* *}$ & $27.9(24 / 86)^{* *}$ & $0(0 / 33)+\dagger$ \\
Associated autoimmune disorders & $0(0 / 54)$ & $35.2(19 / 54)^{* *}$ & $22.1(19 / 86)^{* *}$ & $69.7(23 / 33)^{* *}+\dagger$ \\
Pancreatic mass & $0(0 / 54)$ & $24.1(13 / 54)^{* *}$ & $2.4(2 / 86)^{* *} \dagger$ & $0(0 / 33)+\dagger$ \\
Calcifications & $0(0 / 54)$ & $44.4(24 / 54)^{* *}$ & $82.6(71 / 86)^{* *}+\dagger$ & $0(0 / 33)+\dagger$ \\
Pseudocysts & $0(0 / 54)$ & $16.7(9 / 54)^{* *}$ & $49.4(42 / 85)^{* \star}+\dagger$ & $0(0 / 33) \dagger$ \\
Eosinophilia & $5.6(3 / 54)$ & $13.0(7 / 54)$ & $12.8(11 / 86)$ & $0(0 / 33) \dagger$ \\
ANA & $7.4(4 / 54)$ & $13(7 / 54)$ & $10.5(9 / 86)$ & $51.5(17 / 33)^{* *}+\dagger$ \\
Antimitochondrial Ab & $0(0 / 54)$ & $3.7(2 / 54)$ & $1.2(1 / 86)$ & $6.1(2 / 33)$ \\
Antismooth muscle Ab & $1.9(1 / 54)$ & $5.6(3 / 54)$ & $7.1(6 / 85)$ & $3(1 / 33)$ \\
SS-B & $0(0 / 54)$ & $1.9(1 / 54)$ & $1.2(1 / 86)$ & $39.4(13 / 33)^{* *}+\dagger$ \\
\hline
\end{tabular}

Results are expressed as percentage (number of positive cases/total number of cases).

$\mathrm{CP}$, chronic pancreatitis; ICP, idiopathic chronic pancreatitis; ANA, antinuclear antibodies; $A b$, antibodies; Eosinophilia, $>350 \times 10^{9}$ eosinophils/l.

${ }^{*} \mathrm{p}<0.05,{ }^{* *} \mathrm{p}<0.01$ versus controls; $\mathrm{tp}<0.05,+\uparrow \mathrm{p}<0.01$ versus ICP.

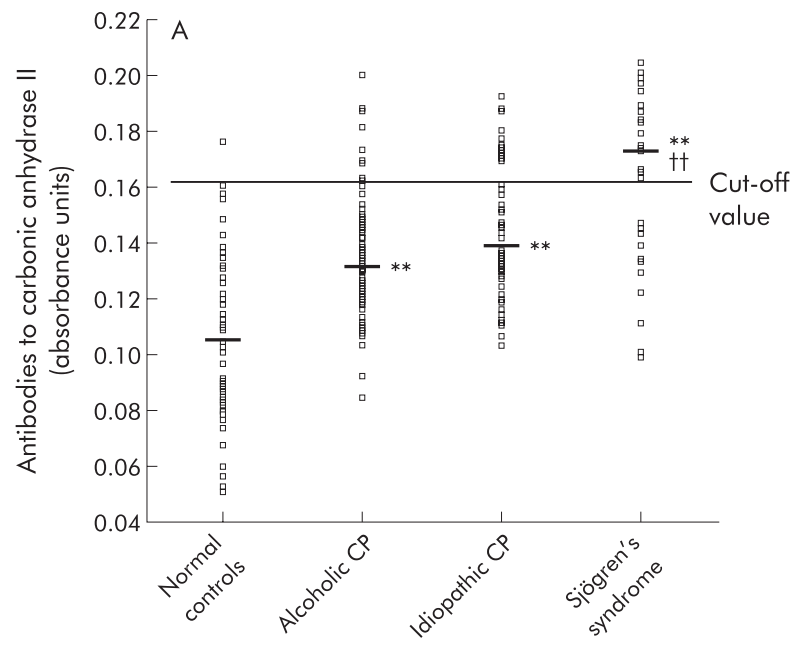

pancreatitis, cholestasis, or jaundice but showed a significantly higher proportion of associated autoimmune disorders and ANA than those with CP.

CA-II Ab in normal subjects and patients with CP or SS Serum levels of CA-II Ab were significantly higher in patients with alcoholic CP, ICP, or SS than in normal controls (fig lA). CA-II Ab levels were also higher in patients with SS than in those with ICP. The percentage of cases with elevated CA-II $\mathrm{Ab}$ was significantly lower $(\mathrm{p}<0.01)$ in normal controls (1.9\%) and in patients with alcoholic CP (10.5\%) compared with patients with ICP $(27.8 \%)$ or SS $(63.6 \%)$. Increased CA-II Ab serum levels were confirmed by western blotting in patients with ICP.

Total IgG and IgG4 in serum from normal subjects and patients with CP or SS

Serum levels of total IgG were significantly higher $(p<0.01)$ in patients with alcoholic or idiopathic CP or with SS than in normal controls. The percentage of cases with elevated levels of IgG was significantly higher $(\mathrm{p}<0.05)$ in patients with alcoholic CP $(36 \%)$, ICP $(42.6 \%)$, or SS $(51.5 \%)$ than in normal controls $(20 \%)$.

An important percentage of patients with ICP (14.8\%; 8/54) exhibited elevated IgG4 levels (fig 1B). The percentage of cases with elevated IgG4 levels was higher $(p<0.05)$ in patients with ICP $(14.8 \%)$ than in patients with SS $(0 \%)$. Serum levels of IgG4 were significantly higher in patients with alcoholic CP than in normal controls although only $8.1 \%$ (7/86) of alcoholic patients had increased IgG4 levels.

\section{Association between CA-II Ab and IgG4 serum levels in patients with ICP}

Among the 54 ICP patients, $13 \%$ had a simultaneous increase in CA-II Ab and IgG4 levels while $14.8 \%$ had increased CA-II $\mathrm{Ab}$ levels but normal IgG4 levels. The proportion of cases with elevated IgG4 serum levels in the ICP group were significantly higher $(\mathrm{p}<0.0001)$ among patients with increased $\mathrm{CA}-\mathrm{II} \mathrm{Ab}$ than in those with normal values (fig $2 \mathrm{~A}$ ). Most patients (7/8) with increased IgG4 levels also exhibited elevated CA-II Ab levels.

Figure 1 Serum levels of antibodies against carbonic anhydrase II (A) and lgG4 levels (B) in normal controls, in patients with alcoholic or idiopathic chronic pancreatitis (CP), and in patients with Sjögren's syndrome. Median values are indicated. The normal range was 700$1200 \mathrm{mg} / \mathrm{dl}$ for serum lgG levels and $60-130 \mathrm{mg} / \mathrm{dl}$ for lgG4 levels. Statistical significance is indicated as follows: ${ }^{* *} p<0.01$ versus healthy subjects; ††p $<0.01$ versus patients with idiopathic pancreatitis.

\section{Increased $C A-I I A b$ and $\lg G 4$ in patients likely to have ACP}

Table 3 shows the associations between increased serum CA-II Ab or elevated serum IgG4 levels and the relevant parameters related to a diagnosis of ACP in the group of patients with ICP. 

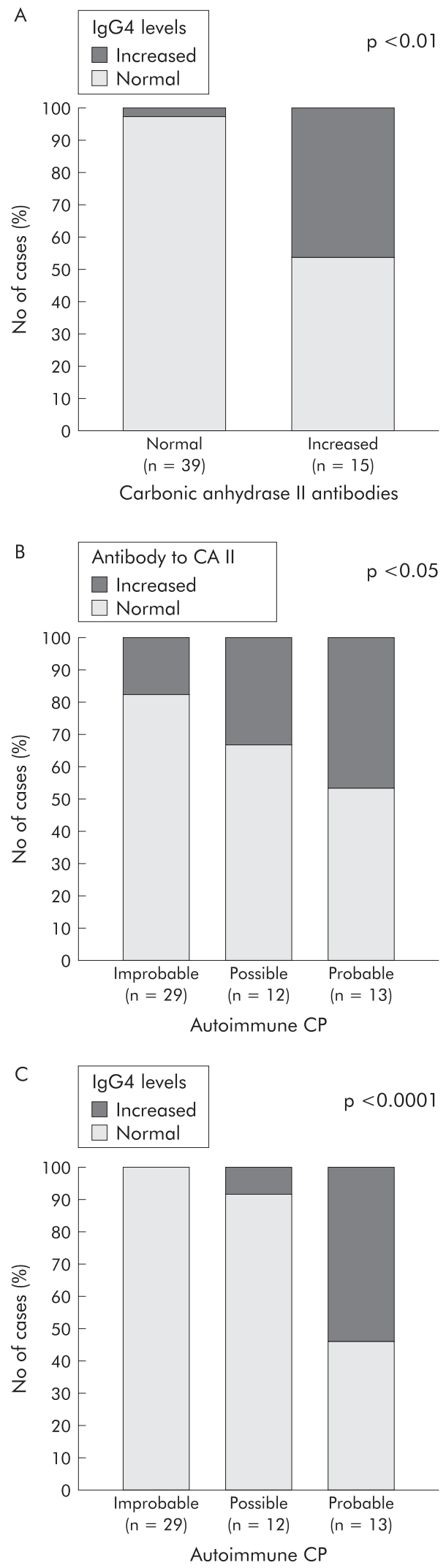

Figure 2 (A) Association between antibodies to carbonic anhydrase II and lgG4 levels in patients with idiopathic chronic pancreatitis (ICP). Association between a diagnosis compatible with autoimmune pancreatitis in ICP patients based on the proposed diagnostic score and antibodies to carbonic anhydrase II (B) or lgG4 levels (C). The p value for statistical significance is shown $\left(\chi^{2}\right.$ of Pearson $(A, C)$ and $\chi^{2}$ for linear trend (B)).
Table 3 Association between antibodies to carbonic anhydrase II (CA-II Ab), IgG4, clinical, morphological, and biochemical parameters in patients with idiopathic chronic pancreatitis

\begin{tabular}{|c|c|c|}
\hline & $\begin{array}{l}\text { Increased } \\
\text { CA-II Ab\% } \\
(n=15)\end{array}$ & $\begin{array}{l}\text { Increased } \\
\text { lgG4\% } \\
(\mathrm{n}=8)\end{array}$ \\
\hline Age & $59(15-83)$ & $59(26-78)$ \\
\hline $\operatorname{Sex}(F / M)$ & $7 / 8$ & $2 / 6$ \\
\hline $\begin{array}{l}\text { Absence of acute attacks of } \\
\text { pancreatitis }\end{array}$ & 80 & 87.5 \\
\hline Cholestasis/jaundice & 33.3 & $75^{* *}$ \\
\hline Associated autoimmune disorders & $60^{*}$ & $87.5^{\star *}$ \\
\hline Pancreatic mass & 46.7 & 62.5 \\
\hline Absence of calcifications & 73.3 & $100^{* *}$ \\
\hline Absence of pseudocysts & 93.3 & 100 \\
\hline $\begin{array}{l}\text { Irregular narrowing of pancreatic } \\
\text { duct }\end{array}$ & 50 & $87.5^{\star *}$ \\
\hline $\lg G(>1200 \mathrm{mg} / \mathrm{dl})$ & $66.7^{*}$ & $100^{* *}$ \\
\hline Antinuclear antibodies & 20 & 25 \\
\hline
\end{tabular}

Our results indicate that most cases with increased CA-II $\mathrm{Ab}$ exhibited clinical and morphological parameters compatible with ACP. Thus a significant association was found between increased serum CA-II Ab levels and elevated serum IgG levels or associated autoimmune disorders in the ICP group. The majority of patients (10/15) with elevated CA-II $\mathrm{Ab}$ levels also had increased IgG levels (table 3). Acute attacks of pancreatitis were absent in most (80\%) ICP patients with increased CA-II Ab but the absence of abdominal pain only occurred in $47 \%$ of these patients. Indeed, pancreatic pain that occurs late in the evolution of CP may not be associated with an increase in serum pancreatic enzymes.

On the other hand, most cases with elevated IgG4 levels also exhibited clinical and morphological parameters compatible with ACP. Thus a significant association was found between IgG4 and cholestasis/jaundice, associated autoimmune disorders, absence of calcifications, irregular narrowing of the pancreatic duct, or increased IgG (table 3). Indeed, all
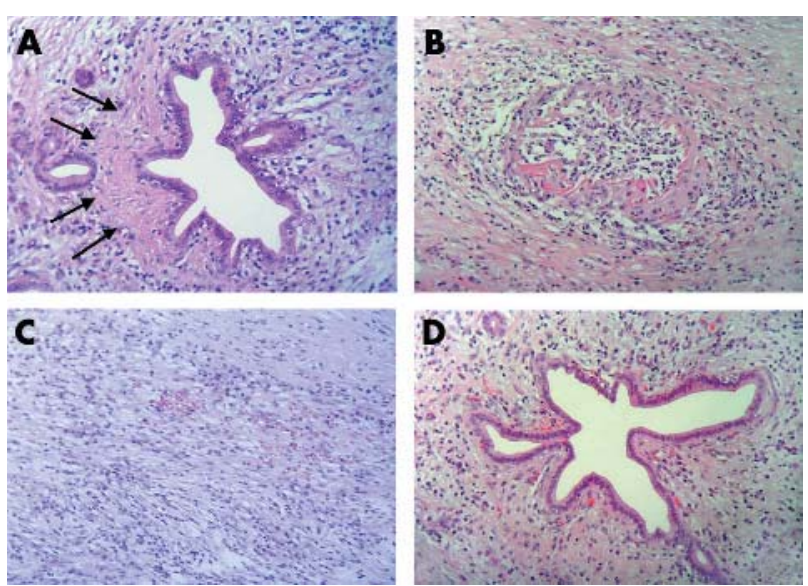

Figure 3 Representative histological images. (A) Pancreatic duct surrounded by a prominent lymphoplasmacytic infiltrate and collagen deposits (arrows). (B) Pancreatic vein showing dense lymphoplamacytic infiltrate and perivenular fibrosis (haematoxylin-eosin, $\times 200$ for all images). (A) and (B) correspond to case No 1 in table 4. (C) Prominent interstitial fibrosis and diffuse lymphoplamacytic infiltrate, which corresponds to patient No 3 in table 4. (D) Pancreatic duct surrounded by a mononuclear lymphoplamacytic infiltrate which corresponds to patient No 4 in table 4 . 
ICP patients with elevated IgG4 exhibited increased IgG but absence of calcifications or pseudocysts. It is worth noting that most of these patients (7/8) exhibited irregular narrowing of the main pancreatic duct, diffuse enlargement of the pancreas, absence of acute attacks of pancreatitis, and associated autoimmune disorders. At least one of the following autoimmune diseases was present in these patients: rheumatoid arthritis, bronchial asthma, sialoadenitis, sacroiliitis, Crohn's disease, and sclerosing cholangitis. Abdominal pain was absent in $50 \%$ of ICP patients with increased IgG4.

When the score previously established in the methods section for a diagnosis of ACP was applied to patients suffering from ICP, we found $24.1 \%$ (13/54) with "probable $\mathrm{ACP}^{\prime}, 22.2 \%$ (12/54) were considered as "possible ACP" whereas $53.7 \%(29 / 54)$ were considered "probably not ACP".

We found a significant association $(\mathrm{p}=0.049)$ between increased serum CA-II Ab and a diagnosis compatible with ACP (fig 2B). CA-II Ab increased in six patients (46.2\%) with "probable ACP", in four patients (33.3\%) with "possible ACP" and in five patients (17.2\%) with "probably not ACP". Accordingly, CA-II Ab was raised in the subgroup likely to have ACP.

Figure 2C shows the marked significant association between IgG4 $(\mathrm{p}<0.0001)$ and the proposed diagnosis of ACP according to the score previously established in the methods section. Taking into account the score for the 54 ICP patients, serum IgG4 levels increased in seven patients $(53.8 \%)$ with "probable $\mathrm{ACP}^{\prime}$, in one patient (8.3\%) with "possible $\mathrm{ACP}^{\prime}$, and in none of the patients $(0 \%)$ with "probably not $\mathrm{ACP}^{\prime}$. It is noteworthy that IgG4 levels were markedly higher $(\mathrm{p}=0.01)$ in patients with "probable ACP" (median 1600 (5th, 90th percentiles 97, 8486)) than in those with "possible ACP" $(253$ (85, 1931)) and those with "probably not $\mathrm{ACP}^{\prime}(241(17,714))$.

When the score established for the diagnosis of ACP was applied to patients suffering from alcoholic CP, only $1.2 \%$ ( $1 / 86)$ were considered "probable ACP", 8.1\% (7/86) "possible ACP" whereas $90.7 \%$ (78/86) were considered "probably not $\mathrm{ACP}^{\prime \prime}$. These results are markedly different from those obtained in patients with ICP. Furthermore, we found no significant association between increased CA-II Ab or IgG4 levels and a diagnosis compatible with ACP in patients suffering from alcoholic CP.

A hierarchical multivariate cluster analysis was also performed to identify patients with a compatible diagnosis of ACP among those initially diagnosed as ICP. When the same clinical, morphological, and biochemical parameters used for the score (acute pancreatitis, abdominal pain, cholestasis/jaundice, autoimmune disorders, pancreatic mass, pseudocysts, calcifications, IgG, and ANA) were included in a first cluster analysis of the 54 ICP patients, a group composed of 25 cases was identified as compatible with ACP. When the
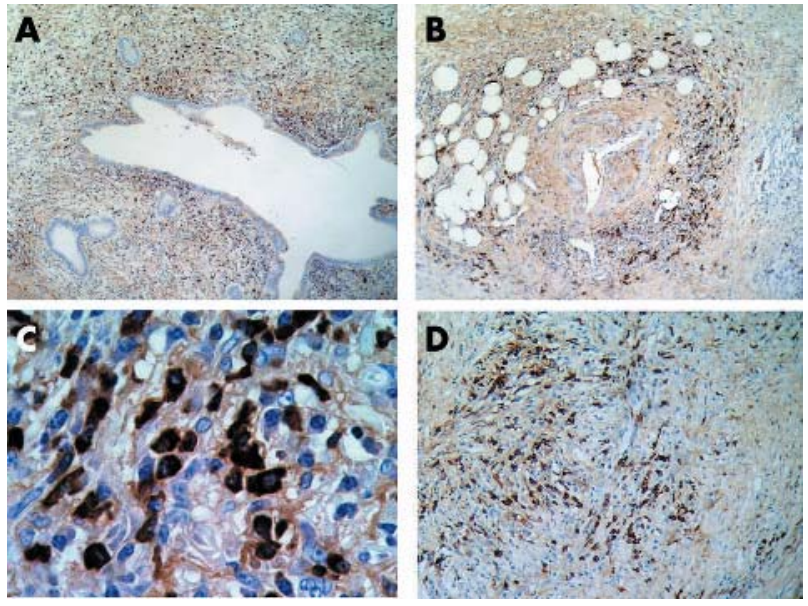

Figure 4 Representative immunohistochemical images with lgG4 immunostaining. (A) Pancreatic duct surrounded by a severe lymphoplasmacytic infiltrate predominantly of $\lg G 4$ positive plasma cells. (B) Prominent lgG4 positive plasma cells within a dense lymphoplamacytic infiltrate surrounding a pancreatic artery. $(A)$ and $(B)$ correspond to case No 1 in table 4. (C) Abundant lgG4 positive plasma cells together with some lgG4 negative plasma cells, which corresponds to patient No 3 in table 4. (D) Diffuse fibrosis around a vein with severe mononuclear lymphoplamacytic infiltrate containing abundant lgG4 positive plasma cells, which corresponds to patient No 4 in table 4 . Amplifications were: $\times 40$ for $A$ and $B, \times 1000$ for $C$, and $\times 200$ for $D$.

increase in CA-II Ab and IgG4 levels was used together with the previous nine parameters in a second cluster analysis, we only found eight cases compatible with ACP. It is worth noting that all of these eight cases were considered as "probable ACP" according to the proposed score. A significant association $(\mathrm{p}<0.0001)$ between both cluster analysis and the score was found.

\section{Increased CA-II $A b$ and IgG4 levels and infiltration of IgG4 positive plasma cells in cases of ACP confirmed by histological analysis}

When surgical specimens from the pancreas were available, histopathological analysis was performed to obtain a definitive diagnosis of ACP if fibrosis and lymphoplasmacytic infiltration were found. Figure 3 shows representative images for the inflammatory infiltrate in four cases of ACP. Table 4 reports the major features of those ACP cases studied by histological analysis. It is worth noting that all four cases with lymphoplasmacytic infiltrate exhibited increased CA-II $\mathrm{Ab}$ and IgG4 levels. Furthermore, immunohistochemical analysis revealed a prominent infiltrate of IgG4 positive

Table 4 Clinical, biological, and histological data in patients operated on with idiopathic chronic pancreatitis

\begin{tabular}{|c|c|c|c|c|c|c|c|c|c|c|c|c|c|c|c|c|c|}
\hline $\begin{array}{l}\text { Patient } \\
\text { No }\end{array}$ & $\begin{array}{l}\text { Age } \\
|y| / \\
\text { sex }\end{array}$ & AP & Pain & $\begin{array}{l}\text { Cholestasis/ } \\
\text { jaundice }\end{array}$ & $\begin{array}{l}\text { Autoimmune } \\
\text { disorders }\end{array}$ & Calcification & Pseudocysts & Mass & Eosinophilia & ANA & $\lg G$ & $\lg G 4$ & $\begin{array}{l}\text { CA } \\
\text { II-Ab }\end{array}$ & Score & Cluster & $\begin{array}{l}\text { Lymphocytic } \\
\text { infiltrate }\end{array}$ & $\begin{array}{l}\text { Plasmacytic } \\
\text { infiltrate }\end{array}$ \\
\hline 1 & $59 / M$ & $N$ & $N$ & $Y$ & Y & $N$ & $N$ & $Y$ & $\mathrm{~N}$ & $N$ & + & + & + & 3 & 2 & + & + \\
\hline 2 & $59 / \mathrm{W}$ & $N$ & $Y$ & $Y$ & Y & $\mathrm{N}$ & $\mathrm{N}$ & $\mathrm{N}$ & Y & $Y$ & + & + & + & 3 & 2 & + & + \\
\hline 3 & $77 / M$ & $N$ & $\mathrm{~N}$ & $Y$ & Y & $\mathrm{N}$ & $N$ & $Y$ & Y & Y & + & + & + & 3 & 2 & + & + \\
\hline 4 & $52 / \mathrm{W}$ & $N$ & $Y$ & $Y$ & Y & $\mathrm{N}$ & $\mathrm{N}$ & Y & Y & $\mathrm{N}$ & + & + & + & 3 & 2 & + & + \\
\hline 5 & $13 / \mathrm{W}$ & $Y$ & $Y$ & $\mathrm{~N}$ & Y & $Y$ & $Y$ & $N$ & $N$ & $Y$ & + & - & - & 1 & 1 & - & - \\
\hline 6 & $62 / \mathrm{W}$ & $N$ & $\mathrm{~N}$ & $\mathrm{~N}$ & $\mathrm{~N}$ & $\mathrm{~N}$ & $\mathrm{~N}$ & $Y$ & $\mathrm{~N}$ & $\mathrm{~N}$ & + & - & - & 3 & 1 & - & - \\
\hline 7 & $37 / M$ & $N$ & $\mathrm{~N}$ & $\mathrm{~N}$ & $\mathrm{~N}$ & $\mathrm{~N}$ & $N$ & Y & $N$ & $\mathrm{~N}$ & + & - & - & 3 & 1 & + & - \\
\hline 8 & $56 / M$ & $N$ & $\mathrm{~N}$ & $Y$ & $\mathrm{~N}$ & $\mathrm{~N}$ & $\mathrm{~N}$ & Y & $\mathrm{N}$ & $\mathrm{N}$ & - & - & - & 2 & 1 & + & - \\
\hline 9 & $37 / W$ & $N$ & $Y$ & $Y$ & $N$ & $Y$ & $\mathrm{~N}$ & Y & $\mathrm{N}$ & $\mathrm{N}$ & - & - & - & 2 & 1 & - & - \\
\hline 10 & $68 / M$ & $Y$ & $Y$ & $\mathrm{~N}$ & $\mathrm{~N}$ & Y & $Y$ & $\mathrm{~N}$ & $\mathrm{~N}$ & $\mathrm{~N}$ & - & - & - & 0 & 1 & - & - \\
\hline
\end{tabular}

AP, acute pancreatitis; ANA, antinuclear antibodies; + , increased antibody or presence of infiltrate; --, absence of increased antibody or infiltrate. Score grading: 0 or 1 , probably not autoimmune chronic pancreatitis (ACP); 2 , possible ACP; 3 , probable ACP. Cluster grading: 1, probably not ACP; 2 , probable ACP. 
plasma cells in all four of these cases (fig 4). In addition, two of these cases ( 1 and 2 ) exhibited a positive response to corticosteroid treatment. The four cases with a definitive diagnosis of ACP were among the eight classified as compatible with ACP by the proposed score and cluster analysis (table 4). Surgical specimens were not available in the remaining four positive cases for ACP found with the cluster analysis.

\section{DISCUSSION}

Since the first description of non-alcoholic primary inflammatory sclerosis of the pancreas by Sarles and colleagues ${ }^{10}$ in 1961, the diagnosis of ACP was hindered by the absence of histological analysis in the majority of cases. Clinically, ACP shows few acute bouts of pancreatitis, absence of calcifications and pseudocysts, but frequent jaundice and pancreatic mass, thus making differential diagnosis with pancreatic cancer difficult. ${ }^{12-15} 20$ In agreement with previous studies, ${ }^{21}$ we found a lower percentage of cases with acute attacks of pancreatitis, calcifications, and pseudocysts in patients with ICP — which included ACP - than in those with alcoholic CP (table 2). However, the percentage of cases with pancreatic mass was remarkably higher in patients with ICP than in those with alcoholic CP.

There is still controversy concerning the definitive diagnosis of ACP. ${ }^{15}$ Recently, the Japan Pancreas Society ${ }^{15}$ proposed diagnostic criteria for ACP that include the presence of autoantibodies, such as CA-II Ab or ANA. ACP responds to corticosteroid treatment and is often associated with other autoimmune diseases. ${ }^{13} 1522$ Hamano and colleagues ${ }^{14}$ reported that an increase in serum IgG4 may distinguish ACP from other diseases of the pancreas. Nevertheless, according to Frulloni and Cavallini, ${ }^{15}$ most of these criteria are not specific for the disease.

By studying 33 patients with ICP, Kino-Ohsaki and colleagues $^{3}$ found increased CA- II Ab in 33\% of cases. Our results show that CA-II Ab increased in $31 \%$ of ICP patients and that this increase was significantly associated with manifestations compatible with ACP (table 3). Nevertheless, the increase in serum CA-II Ab occurred not only in patients with SS (64\%) - as a positive control group for CA-II Ab-or ICP (31\%) but also in those with alcoholic CP (10\%) (fig 2B), in agreement with other studies. ${ }^{323}$ Consequently, the rise in serum CA-II Ab is not specific for ACP. However, the percentage of our cases with elevated CA-II Ab was significantly higher in the ICP group than in the group with alcoholic CP.

A simplified score of easy clinical use was proposed in this study to assess the major clinical, morphological, and biochemical parameters-except IgG4 and CA-II Ab-of relevance for a diagnosis of ACP. A significant association was found between increased CA-II Ab and a diagnosis compatible with ACP-46\% of "probable ACP" and 33\% of "possible ACP (fig 2 B). Therefore, CA-II Ab are elevated in patients likely to have ACP.

In the present study, serum IgG4 levels increased over the established cut off value in some patients with chronic pancreatitis, either idiopathic (14.8\%) or alcoholic $(8.1 \%)$. Consequently, the increase in IgG4 levels does not seem specific for ACP. However, it is worth noting that four of eight ICP patients with increased IgG4 exhibited very high serum IgG4 levels ( $>2$-fold the cut off value) and this marked increase was not found in any patient with alcoholic CP. In accordance with Hamano and colleagues, ${ }^{14}$ the increase in IgG4 did not occur in our patients with SS. These results support the hypothesis that IgG4 may serve to identify two immunological types of autoimmune exocrinopathies, one with normal IgG4 levels, such as SS, and another with elevated IgG4 levels, such as ACP. ${ }^{14}{ }^{15}$ This latter form should not be considered as primary autoimmune pancreatitis as most of our idiopathic cases (7/8) with increased IgG4 levels were associated with other autoimmune disorders, such as sclerosing cholangitis, ulcerative colitis, and lupus erythematosus.

The increase in serum IgG4 levels was strongly associated with elevated CA-II levels in ICP patients (fig 2A) and manifestations compatible with ACP (table 3). A marked association was also found between increased IgG4 and a diagnosis compatible with ACP according to the score-54\% of "probable ACP" and 8\% of "possible $\mathrm{ACP}^{\prime}$ ( fig 2C). It is noteworthy that no increase in IgG4 was found among those cases of "probably not ACP". This statistical association was confirmed by a hierarchical multivariate cluster analysis. These results strengthen the relevance of $\operatorname{IgG} 4$ in the diagnosis of ACP. To our knowledge, this is the first report on the close relationship between IgG4, CA-II Ab, and manifestations compatible with ACP.

Klöppel and colleagues ${ }^{20}$ have emphasised the importance of an inflammatory mass in the head of the pancreas and the histological hallmarks for a definitive diagnosis of ACP. According to these authors, fibrotic changes occupy extensive areas compatible with an inflammatory tumour. In addition, there is variability of lesions according to the severity of the disease and status of development, from lymphoplasmacytic infiltrate primarily subepithelial focused on the ducts to involvement of the acinar parenchyma in severe cases. ${ }^{20}$ More recently, lymphoplasmacytic sclerosing pancreatitis was described by Notohara and colleagues ${ }^{24}$ showing elderly male preponderance, pancreatic swelling, and irregular narrowing of the main pancreatic duct.

We reported here four cases with advanced ACP that exhibited prominent fibrosis and lymphoplasmacytic infiltrate, ducts with starlike structure, perivenulitis, and involvement of the acinar parenchyma (fig 3). It is worth noting that all four of these cases (patient Nos $1-4$ in table 4 ) exhibited an increase in both serum IgG4 and CA-II Ab levels and were considered as "probable ACP" according to the proposed score and cluster analysis (table 4). They were associated with other autoimmune disorders and accordingly, IgG4 levels cannot differentiate primary from secondary ACP, as recently suggested. ${ }^{15}$ An intense infiltrate of IgG4 positive plasma cells-characteristic of ACP, as reported by Kamisawa and colleagues ${ }^{19}$ - was found in these four cases (fig 4).

Lymphocytic, but not plasmacytic, infiltrate was found in two cases (patient Nos 7 and 8 in table 4) with a diagnosis compatible with ACP but not elevated IgG4 or CA-II Ab levels. Whether these two cases represent different stages in the evolution of ACP without production of autoantibodies or infiltration of activated lymphocytes remains to be established. Infiltrate of plasma cells was only found in those cases with increased serum IgG4 and CA-II Ab levels. Therefore, the presence of both elevated IgG4 and CA-II Ab was associated with a definitive diagnosis of ACP.

Based on the results obtained in this study, we propose the following algorithm as a useful tool for the diagnosis of ACP in patients with an initial diagnosis of ICP. Firstly, use the proposed score that assesses major clinical, morphological, and biochemical parameters relevant for the ACP diagnosis (see methods section). Secondly, if the score provides a compatible diagnosis of ACP-either "probable" or "possible"-then measure serum IgG4 and CA-II Ab levels (the latter by ELISA or western blotting). Thirdly, if both IgG4 and CA-II Ab levels are increased, then analyse the pancreatic duct by ERCP imaging and perform histological analysis, or alternatively, test the response to corticosteroid treatment.

In summary, the results of this multicentre study indicate that the increase in serum IgG4 levels is strongly associated with elevated CA-II Ab and manifestations compatible with ACP. A definitive diagnosis of ACP by histological analysis was associated with other autoimmune disorders and an increase in both serum IgG4 and CA-II Ab levels. A simplified 
score of easy clinical use together with an algorithm for the diagnosis of ACP have been proposed.

\section{ACKNOWLEDGEMENTS}

We thank Dr Manuel Castelho, Hospital Militar of Valencia, for his help with the immunoglobulin assays and Centro de Transfusiones de la Comunidad Valenciana (Spain) for providing blood samples from healthy donors. We also thank Mrs Remedios Gonzalez for her technical help and Mrs Marilyn Noyes for revising the manuscript.

\section{Authors' affiliations}

L Aparisi, Unit of Hepatology, Universitary Clinic Hospital, Valencia, Spain A Farre, Service of Gastroenterology, Santa Creu and Sant Pau Hospital, Barcelona, Spain

L Gomez-Cambronero, S Mery, J Sastre, Department of Physiology,

School of Medicine, University of Valencia, Spain

J Martinez, M Perez-Mateo, Department of Gastroenterology, University General Hospital, Alicante, Spain

G De Las Heras, Department of Gastroenterology, Marqués de Valdecilla Hospital, Santander, Spain

J Corts, Department of Rheumatology, Universitary Clinic Hospital,

Valencia, Spain

S Navarro, Department of Gastroenterology, Hospital Clinic and Institut $\mathrm{d}^{\prime}$ Investigacions Biomèdiques August Pi i Sunyer (IDIBAPS), Barcelona, Spain

J Mora, Department of Biochemistry, Santa Creu and Sant Pau Hospital, Barcelona, Spain

M Lopez-Hoyos, Department of Immunology, Marqués de Valdecilla Hospital, Santander, Spain

L Sabater, Department of Surgery, Universitary Clinic Hospital, Valencia, Spain

A Ferrandez, Department of Pathology, School of Medicine, University of Valencia, Spain

D Bautista, Department of Preventive Medicine, Hospital Dr Peset,

University of Valencia, Spain

Conflict of interest: None declared.

\section{REFERENCES}

1 Strand V, Talal N. Advances in the diagnosis and concept of Siögren's syndrome (autoimmune exocrinopathy). Bull Rheum Dis 1980;30:1046-52.

2 Epstein O, Chapman R, Kaje-Bakaar G, et al. The pancreas in primary biliary cirrhosis and primary sclerosing cholangitis. Gastroenterology 1982;83:1177-82.

3 Kino-Ohsaki J, Nishimori I, Morita M, et al. Serum antibodies to carbonic anhydrase I and II in patiens with idiopatic chronic pancretitis and Sjögren's syndrome. Gastroenteroloy 1996;110:1579-86.
4 Inagaki $Y$, Jinno-Yoshida $Y$, Hamasaki $Y$, et al. A novel autoantiboy reactive with carbonic anhydrase in sera from patients with systemic lupus erythematosus and Sjögren's syndrome. J Dermatol Sci 1991;2:147-54.

5 Itoh Y, Reichlin M. Antibodies to carbonic anhydrase in systemic lupus erithematosus and other rheumatic diseases. Arthritis Rheum 1992;35:73-82.

6 Gordon SC, Quattrociocchi-Longe TM, Khan BA, et al. Antibodies to carbonic anhydrase in patients with immune cholangiopathies. Gastroenterology 1995; 108:1802-9.

7 Nishimori I, Bratanova T, Toshkov I, et al. Induction of experimental autoinmune sialoadenitis by inmunization of $\mathrm{PL} / \mathrm{J}$ mice with carbonic anhidrase II. J Immunol 1995;154:4865-73.

8 Ueno Y, Motoyasu I, Takahashi S, et al. Different susceptibility of mice to immune-mediated cholangitis induced by immunization with carbonic anhydrase II. Lab Invest 1998;78:629-37.

9 Uchida K, Okazaki K, Nishi T, et al. Experimental immune-mediated pancreatitis in neonatally thymectomized immunized with carbonic anhydrase II and lactoferrin. Lab Invest 2002;82:411-24.

10 Sarles H, Sarles JC, Muratore R, et al. Chronic inflammatory sclerosis of the pancreas - an autonomous pancreatic disease? Am J Dig Dis 1961;6:688-98.

11 Yoshida K, Toki F, Takenchi T, et al. Chronic pancreatitis caused by an autoinmune abnormality. Proposal of the concept of autoinmune pancreatitis. Dig Dis Sci 1995;40:1561-8.

12 Wakabayashi T, Motoo Y, Kojima Y, et al. Chronic pancreatitis and diffuse irregular narrowing of the main pancreatic duct. Dig Dis Sci 1998;43:2415-25.

13 Erkelens GW, Vleggaar FP, Lesterhuis W, et al. Sclerosing pancreatocholangitis responsive to steroid therapy. Lancet 1999;354:43-4.

14 Hamano H, Kawa S, Hoiuchi A, et al. High serum lgG4 concentrations in patients with sclerosing pancreatitis. N Engl J Med 2001;344:732-8.

15 Steinberg WM, Barkin JS, Bradley EL, et al. Controversies in clinical pancreatology. Autoimmune pancreatitis: does it exists, Pancreas 2003;27:1-13.

16 Sarner M, Cotton P. Classification of pancreatitis. Gut 1984;25:756-9.

17 Singer M, Gyr K, Sarles H. Revised classification of pancreatitis. Gastroenterology 1985;89:682-5.

18 Vitali C, Bombardieri S, Motsououlos H, et al. Preliminary criteria for the classification of Sï̈gren's syndrome: Results of a prospective concerted action supported by the European Community. Arthritis Rheum 1993;36:340-7.

19 Kamisawa T, Funata N, Hayashi Y, et al. Close relationship between autoimmune pancreatitis and multifocal fibrosclerosis. Gut 2003;52:683-7. 20 Klöppel G, Lüttges J, Löhr M, et al. Autoimmune pancreatitis: Pathological, clinical, and immunological features. Pancreas 2003;27:14-19.

21 Layer $\mathbf{P}$, Yamamoto $H$, Kalthoff $H$, et al. The different courses of early- and late-onset idiopathic and alcoholic chronic pancreatitis. Gastroenterology 1994; 104:1481-7.

22 Ito T, Nakano I, Koyanagi S, et al. Autoimmune pancreatitis as a new clinical entity: Three cases of autoimmune pancreatitis with effective steroid therapy. Dig Dis Sci 1997;42:1458-68.

23 Bartolome MJ, De las Heras G, López-Hoyos M. Low-avidity antibodies to carbonic anhydrase-I and $-I I$ in autoimmune chronic pancreatitis. Sci World J 2002;2:1560-8.

24 Notohara K, Burgart $\sqcup$, Yadav D, et al. Idiopathic chronic pancreatitis with periductal lymphoplasmacytic infiltration. Am J Surg Pathol
2003;27:1119-27.

\section{EDITOR'S QUIZ: GI SNAPSHOT}

\section{Answer}

From question on page 672

As fig 1 shows, there is a large complex shunt present joining a portal vein (PV) and an adjacent hepatic vein (HV) (left). There is focal necrosis of the parenchyma (top left). The right figure shows hepatic vein $0.5 \mathrm{~mm}$ in diameter shows luminal obliteration by organised thrombosis. There is severe necrosis of the surrounding liver parenchyma (both $52 \times$, elastic trichrome stain).

The resected liver contained many arteriovenous malformations compatible with hereditary haemorrhagic telangiectasia (Osler-Weber-Rendu disease). In addition, we found ischaemic necrosis and organising hepatic vein thrombosis involving the medium and small veins.

Pregnancy is a risk factor for hepatic vein thrombosis because of enhanced coagulability and compression by the uterus on the vena cava and liver. In the present case, pre-existent arteriovenous malformations may have made the hepatic veins more susceptible to thrombosis because of turbulent flow. Hypovolaemic shock could also have contributed to thrombus formation and hepatic necrosis. The different ages of necrosis and thrombosis in the present liver suggest a stepwise progression of the ischaemic change. Hepatic vein thrombosis leading to lobular necrosis and hepatomegaly probably began about three weeks prior to delivery when she complained of abdominal pain.

The diagnosis of hereditary hemorrhagic telangiectasia was confirmed with the Arg411Trp mutation in exon 8 of the ALKl gene. The disease is inherited as an autosomal dominant trait with an incidence of $1: 2500$. The prevalence of clinical liver involvement is $8-31 \%$. 